Review Article

\title{
Effects of Combined Physical Activity and Cognitive Training on Cognitive Function in Older Adults with Subjective Cognitive Decline: A Systematic Review and Meta-Analysis of Randomized Controlled Trials
}

\author{
Qianqian Sun, ${ }^{1,2}$ Shurui Xu, ${ }^{1,2}$ Shuai Guo, ${ }^{1,2}$ Yue You, ${ }^{1,2}$ Rui Xia, ${ }^{2}$ and Jiao Liu $\mathbb{D}^{1,2,3,4}$ \\ ${ }^{1}$ College of Rehabilitation Medicine, Fujian University of Traditional Chinese Medicine, Fuzhou, Fujian, China \\ ${ }^{2}$ National-Local Joint Engineering Research Center of Rehabilitation Medicine Technology, \\ Fujian University of Traditional Chinese Medicine, Fuzhou, Fujian, China \\ ${ }^{3}$ Traditional Chinese Medicine Rehabilitation Research Center of State Administration of Traditional Chinese Medicine, \\ Fujian University of Traditional Chinese Medicine, Fuzhou, Fujian, China \\ ${ }^{4}$ Key Laboratory of Chinese Medicine Orthopedics and Sports Rehabilitation of Ministry of Education, \\ Fujian University of Traditional Chinese Medicine, Fuzhou, Fujian, China
}

Correspondence should be addressed to Jiao Liu; liujiao0415@outlook.com

Received 20 September 2020; Revised 20 March 2021; Accepted 17 April 2021; Published 29 April 2021

Academic Editor: Robson Xavier Faria

Copyright ( 2021 Qianqian Sun et al. This is an open access article distributed under the Creative Commons Attribution License, which permits unrestricted use, distribution, and reproduction in any medium, provided the original work is properly cited.

Background. Subjective cognitive decline (SCD) is recognized as the earliest prodromal stage of Alzheimer's disease (AD). Emerging studies explored the effects of combined physical activity and cognitive training interventions on cognitive ability, psychological wellbeing, and emotion of older adults with SCD, but the results are now still controversial. Objective. This study systematically evaluated the enhancement effects of the combined physical-cognitive interventions on memory self-efficacy, objective cognitive function, psychological well-being, and emotion of older adults with SCD. Methods. Data sources PubMed, EMBASE, Web of Science, China National Knowledge Infrastructure (CNKI), Wanfang degree and conference papers database, Chinese Science and Technology Periodical (VIP) databases from their inception to 28 February 2020, the Cochrane Central Register of Controlled Trials (Cochrane Library, 2020, Issue 3), and the reference lists of all retrieved articles were searched. Data analysis and bias risk evaluation were conducted in 2020. Two reviewers (SG and YY) independently evaluated the risk of bias of the included studies using the RoB 2 tool. Results. Eleven RCTs involving 1713 participants with SCD (age 68.0 \pm 6.1 ) were included for review and meta-analysis. The interventions in the included studies were physical activity combined with cognitive training. Multiple-modality exercise with mindmotor training, supervised strategy-based memory training sequentially after stationary cycling, Dejian Mind-Body intervention, and physical activity and cognitive stimulation were also practiced. Conclusions. Compared to the active or nonactive control groups, the combined interventions are effective in improving objective cognitive function in SCD which may show the potential value of combined physical-cognitive interventions in improving objective cognitive ability and preventing the conversion of SCD to MCI or $\mathrm{AD}$ and no adverse effects. However, owing to the limitations of the included studies, these findings should be interpreted cautiously.

\section{Introduction}

As the population ages, concerns about cognitive decline increase. 2020 Alzheimer's disease (AD) facts and figures described that total payments are to be $\$ 244$ billion in 2019 for health care, long-term care, and hospice services for people aged $\geq 65$ years with dementia in the United States.
The average per-person Medicare payments for services to beneficiaries of age $\geq 65$ years with (AD) or other dementias are more than three times as great as payments for beneficiaries without these conditions (more than 23 times as great) [1]. AD remains the most common cause of dementia $[1,2]$. Early detection and intervention of Alzheimer's disease offer numerous medical, emotional, and financial 
benefits that accrue to affected individuals and their families as well as to the society at large [3].

Studies have been made to identify individuals who are at increased risk of $\mathrm{AD}$ and to test interventions that might delay the progression of preclinical stage prodromal to fullblown dementia [4-6]. Subjective cognitive decline (SCD) has been identified as a condition where some individuals experience subjective cognitive complaints, but the cognitive performance by neuropsychological tests and daily function shows no evidence of objective cognitive impairment. SCD is increasingly recognized as the earliest prodromal stage of $\mathrm{AD}$ [7-10]. 74\% of people aged 70 years and older who perform normally on standard cognitive tests self-report a subjective decline in cognitive functioning [11]. SCD individuals showing the positivity of pathophysiological $\mathrm{AD}$ biomarkers show a risk of progression similar to MCI patients [6]. Some studies showed that amyloid and tau protein PET imaging investigations in cohorts of aging individuals (>60 years of age) who were cognitively unimpaired showed a quantitative association between the extent of either amyloid or tau pathology with the severity of cognitive concerns experienced by individuals [12-14]. Other studies have investigated markers of neurodegeneration in individuals with SCD. In particular, cross-sectional studies [15-17] have shown that these individuals have small reductions in the volume of brain regions that typically show loss of volume in the early stages of AD (e.g., the medial temporal lobe) when compared with control groups of individuals without SCD. Besides, some studies $[16,18]$ have identified AD-associated patterns of glucose hypometabolism in individuals with SCD, whereas other studies [19] have not. In addition, individuals with SCD who harbor the major genetic risk factor $\mathrm{APOE} \varepsilon 4$ for $\mathrm{AD}$ are at an increased risk of being at individuals with SCD who do not carry the disease-associated APOE $\varepsilon 4$ [20]. It is likely that a relationship between $\mathrm{APOE} \varepsilon 4, \mathrm{SCD}$, and $\mathrm{AD}$ exists, though its exact nature remains undetermined [21].

Livingston et al. considered SCD as a critical window of opportunity to intervene and alter the trajectory of both cognitive and functional decline in seniors [22]. It would be more appropriate to prevent or postpone the AD process by intervention in the SCD stage. However, until now, the positive effect on the cognitive ability and behavioral symptoms of SCD individuals of medication was controversial [23-26]. The available evidence suggests that nonpharmacologic interventions may benefit current cognitive function in persons with SCD [27, 28]. Extensive evidence indicates that physical activity may improve cognitive health outcomes such as mental health, physical health, and quality of life for older adults with SCD. Tailored physical activity guidelines are needed to support strategies for dementia risk reduction [29]. Emerging reviews of the evidence have led international health organizations, including the World Health Organization (WHO), the American National Institutes of Health, and the British National Institute of Health and Care Excellence, to advocate for physical activity as both a primary prevention (in cognitively healthy individuals) and a secondary prevention (in individuals who have no dementia but already experience cognitive decline) strategy for reducing dementia risk [30-33]. The Canadian Physical Activity Guidelines for adults with SCD also have been developed [34].

Physical activity was associated with brain health benefits, including possible improved cognitive function, reduced anxiety, and depression risk, and improved sleep and quality of life [35]. Recent meta-analyses of randomized controlled trials show that physical activity also may be a promising intervention in the cognition of included subjects with dementia and mild cognitive impairment (MCI) $[36,37]$. Cross-sectional studies, longitudinal observational studies, and prospective intervention trials also supported this evidence $[38,39]$.

By adding, exposure to a cognitively challenging environment could enhance the neural and cognitive benefits elicited by physical activity in humans and animals [40, 41]. A review indicated that the new generation of prevention trials is addressing some of the problems encountered in previous trials by testing interventions during the optimum window of exposure (i.e., earlier in the course of the disease, even in the preclinical phase), using multidomain rather than single-domain intervention strategies [42]. Some studies observing the synergistic effect of exercise and cognitive training interventions were associated with improved cognitive performance in older adults [43, 44]. A meta-analysis found that combined cognitive and physical exercise training can improve global cognition in older adults with MCI or AD and were beneficial for activities of daily living and mood [45].

However, for the elderly with SCD, combined physicalcognitive interventions on cognitive function are equivocal. The topic of interventions in this population has been relatively unexplored. For instance, compared with an active or nonactive control group, whether combined physical-cognitive interventions prevent cognitive decline is unclear. In the present study, we aimed to systematically evaluate the effect of combined physical-cognitive interventions on memory self-efficacy, objective cognitive function, general psychological well-being, and emotion.

\section{Methods}

2.1. Selection and Identification of Studies. Seven electronic databases, including PubMed, EMBASE, Web of Science (SCI, Science Citation Index), China National Knowledge Infrastructure (CNKI), Wanfang degree and conference papers database, Chinese Science and Technology Periodical (VIP) databases from their inception to 28 February 2020, and the Cochrane Central Register of Controlled Trials (Cochrane Library, 2020, Issue 3), were searched without language restrictions. Relevant keywords related to physical activity as Medical Subject Heading terms and text words (e.g., "Multiple training modalities" or "Physical activity" and "Activity") were used in combination with words related to subjective cognitive decline (e.g., "Subjective Cognitive Decline," "Subjective Cognitive Impairment," "Cognitive complaints," "Subjective Memory Decline," and "Subjective Memory Impairment"). The unpublished/grey literature was searched using ClinicalTrials.gov, the National Research 
Register, the Chinese Master's Theses Full-Text Database, China Doctor Dissertation Full-Text Database, and China Proceedings of Conference Full-Text Database. Additional trials were found through the reference lists of all retrieved articles (please see the full search strategy in Table 1). Literature search, study identification, and data extraction were conducted from February to April 2020. All searched records were imported into the reference management software (Note Express V.3.2) to eliminate duplicate records. The full texts of the studies that potentially met the inclusion criteria were obtained to further evaluate their eligibility. Any disagreements were resolved by discussion with the third reviewer (QS). The review was registered at the International Prospective Register of Systematic Review, PROSPERO (Registration number: CDR42020181482, https://www.crd. york.ac.uk/prospero/).

2.2. Inclusion Criteria. The trials selected in this review met the following inclusion criteria: (1) study design: published randomized controlled trials (RCTs) were included; (2) participants should older adults (age: 55 years and older) with self-reported cognitive complaints, without objective evidence of deficits on cognitive tests and unaccounted for medical or psychiatric causes (e.g., dementia, MCI); (3) intervention: physical activities (e.g., aerobic activity, muscle-strengthening activity, bone-strengthening activity, balance activity, and multicomponent activity) combined with cognitive training (e.g., cognitive training, memory training, psychological interventions) were practiced in the experimental group for at least 2 weeks; (4) control should be memory intervention, mental training, or health education (e.g., psychoeducation, cognitive restructure, stress management, relaxation, and mindfulness); (5) outcomes: we used subjective memory measures as the primary outcome and objective cognitive function, psychological well-being, and emotion as the second outcomes. Those without available data were excluded.

2.3. Data Extraction. The data were extracted by one reviewer (SX) using the prepared form and checked for accuracy by another reviewer (QS). The extracted information from eligible studies included participants' characteristics, sample size, study design, methodological information of study quality, experimental and control intervention, duration, frequency, intensity and style of aerobic exercise, outcomes, and adverse events.

2.4. Assessment of Risk of Bias in Individual Studies. Two reviewers (SG and YY) independently evaluated the risk of bias of the included studies using the RoB 2 tool, a revised Cochrane risk of bias tool for RCTs, which is a widely used quality assessment tool for evaluating RCTs [46]. The RoB 2 tool includes domains such as randomization process, deviations from intended interventions, missing outcome data, outcome measurement, selection of the reported result, and overall bias [46]. The overall judgment of each item for each study was categorized as "low risk," "high risk," and "some concerns" according to the levels of bias. Discrepancies between reviewers were resolved by the third reviewer (JL).

2.5. Statistical Analysis. Review Manager Software V.5.3 was used for the statistical analysis which was provided by Cochrane Collaboration, and the statistical significance was defined as a two-sided $P$ value $<0.05$. Data were summarized using relative risk with $95 \%$ CI for binary outcomes and mean difference (MD) or standardized MD (SMD) with corresponding 95\% CI for continuous outcomes. The pooled effect was calculated using the fixed-effect model if data were available and no significant heterogeneity was detected. Otherwise, the random-effect model was applied. Statistical heterogeneity was assessed using the $\mathrm{I}^{2}$ statistic. However, the overall pooled analysis was not considered appropriate when the heterogeneity among studies was high $\left(I^{2}>75 \%\right)$; clinical or methodological heterogeneity was considered the potential causes. Heterogeneity among studies was explored using a $\chi^{2}$ test and Higgins $I^{2}$ values [46]. In view of possible heterogeneity between studies, studies with different intervention types were divided into subgroups for analysis according to different factors.

Due to the diversity of the outcome measures related to objective cognitive function in the included studies, and the relatively small number of studies meeting the inclusion criteria, we chose to combine outcomes of global cognitive ability and specific domains of cognition (i.e., memory, attention, executive ability, and verbal fluency) into objective cognitive function. Moderator analyses will be used conducted to explain the variance between groups if the heterogeneity among studies was high $\left(I^{2}>75 \%\right)$.

\section{Results}

3.1. Study Selection. 2100 records were identified from preliminary searches according to the predetermined search strategy. Two reviewers (SX, GS) disregarded obviously irrelevant records based on the abstract or title. A total of 53 potential studies were further evaluated for their eligibility, and 15 studies ultimately fulfilled the inclusion criteria [47-62]. However, 4 studies did not provide the original data $[49,50,60,61]$. We attempted to contact the original authors by e-mail but did not receive any responses. Therefore, we excluded these studies. Finally, 11 studies involving 1713 participants with SCD were included in the review. The detailed literature search and screening flow is presented in Figure 1.

3.2. Study Characteristics. The characteristics of each included study were summarized in Table 1 . Eleven RCTs involving 1713 participants with SCD (age 68.0 \pm 6.1 ) were included for review. The included studies came from Canada (two) [47, 60], Unite States (four) [51, 56, 58, 59], China (one) [52], Australia (two) [53, 57], and France (two) $[54,55]$. Participants were recruited from community $[47,51,55,56]$, social centers [52], social clubs, or personal $[54,58,59]$, memory clinics [57, 60], or hospitals [53]. All included studies reported clear diagnostic inclusion and 
TABLE 1: Characteristics of included studies.

\begin{tabular}{|c|c|c|c|c|c|}
\hline Author/Year & Mean age & $\mathrm{N}(\mathrm{M} / \mathrm{F})$ & Intervention & Frequency, duration and intensity & Outcomes/measure \\
\hline $\begin{array}{l}\text { Boa et al. } \\
2020[47-50]\end{array}$ & $\begin{array}{l}\mathrm{T}: 67.6 \pm 7.5 \\
\mathrm{C}: 67.4 \pm 7.2\end{array}$ & $\begin{array}{c}127 \\
(37 / 90)\end{array}$ & $\begin{array}{l}\text { T: Physical activity } \\
\text { combined cognitive } \\
\text { training } \\
\text { C: Active control }\end{array}$ & $\begin{array}{c}60 \mathrm{~min} / \mathrm{day}, 3 \text { days/week, } 24 \text { weeks } \\
65-85 \% \text { of maximum HR }\end{array}$ & $\begin{array}{l}\text { Working memory/ } \\
\text { Monkey Ladder } \\
\text { Spatial short-term } \\
\text { memory/Spatial Span } \\
\text { Verbal working } \\
\text { memory/Digit Span } \\
\text { Visuospatial paired } \\
\text { associate learning/ } \\
\text { Paired Associates }\end{array}$ \\
\hline $\begin{array}{l}\text { McEwen et al. } 2018 \\
\text { [51] }\end{array}$ & $\begin{array}{l}\mathrm{T}: 65.4 \pm 3.0 \\
\mathrm{C}: 67.0 \pm 5.1\end{array}$ & $55(17 / 38)$ & $\begin{array}{l}\text { T: Memory training } \\
\text { after station cycling } \\
\text { (SEQ) } \\
\text { C: Active control }\end{array}$ & $\begin{array}{c}120 \mathrm{~min} / \text { day, } 2 \text { days/week, } 4 \text { weeks } \\
65 \% \text { HRR }\end{array}$ & $\begin{array}{l}\text { Composite memory/ } \\
\text { Verbal and Visual } \\
\text { Memory Test } \\
\text { Attention/Stroop Test } \\
\text { Executive function/CNS } \\
\text { Processing speed/CNS } \\
\text { Memory/HKLLT }\end{array}$ \\
\hline $\begin{array}{l}\text { Chan et al. } 2017 \\
\text { [52] }\end{array}$ & $\begin{array}{l}\text { T: } 68.3 \pm 4.4 \\
\text { C: } 69.5 \pm 6.9\end{array}$ & $48(12 / 36)$ & $\begin{array}{l}\text { T: Dejian Mind-Body } \\
\text { C: Active control }\end{array}$ & $150 \mathrm{~min} /$ day, 1 days/week, 10 weeks & $\begin{array}{l}\text { Immediate recall/WMS- } \\
\text { III-VR } \\
\text { delayed recall/WMS-III- } \\
\text { VR }\end{array}$ \\
\hline $\begin{array}{l}\text { Lautenschlager et } \\
\text { al. } 2008 \text { [53] }\end{array}$ & $\begin{array}{l}\mathrm{T}: 68.6 \pm 8.7 \\
\mathrm{C}: 68.7 \pm 8.5\end{array}$ & $\begin{array}{c}170 \\
(84 / 86)\end{array}$ & $\begin{array}{l}\text { T: Physical activity } \\
\text { C: Active control }\end{array}$ & $\begin{array}{c}50 \mathrm{~min} / \text { day, } 3 \text { days/week, } 24 \text { weeks } \\
\text { Moderate intensity }\end{array}$ & $\begin{array}{c}\text { Global cognitive } \\
\text { function/CDR, ADAS- } \\
\text { Cog } \\
\text { immediate recall/word } \\
\text { list } \\
\text { delayed recall/word list } \\
\text { Information processing } \\
\text { speed/DSC } \\
\text { verbal fluency/VFT } \\
\text { Depression/BDI } \\
\text { Mentality/ MCS } \\
\text { Physical fitness/ PCS }\end{array}$ \\
\hline $\begin{array}{l}\text { Fabre et al. } 1999 \\
{[54]}\end{array}$ & $\begin{array}{c}\text { AMT: } 64.9 \pm 3.9 \\
\text { AT: } 65.4 \pm 6.2 \\
\text { C: } 67.5 \pm 3.4\end{array}$ & 24 & $\begin{array}{c}\text { AMT: Physical activity } \\
\text { combined cognitive } \\
\text { training } \\
\text { AT: Physical activity } \\
\text { C: Control }\end{array}$ & $90 \mathrm{~min} /$ day, 1 days/week, 2 months & $\begin{array}{c}\text { Memory/WMS } \\
\text { Depression/SQLP } \\
\text { Mentality/SQLP } \\
\text { Physical fitness/SQLP }\end{array}$ \\
\hline $\begin{array}{l}\text { Andrieu et al. } 2017 \\
\text { [55] }\end{array}$ & $\begin{array}{l}\mathrm{T}: 75.0 \pm 4.1 \\
\mathrm{C}: 75.1 \pm 4.3\end{array}$ & $\begin{array}{c}770 \\
(266 / 504)\end{array}$ & $\begin{array}{l}\text { T: Physical activity } \\
\text { combined cognitive } \\
\text { training } \\
\text { C: Control }\end{array}$ & $\begin{array}{l}120 \mathrm{~min} / \text { day, } 2 \text { days/week for the first } \\
\text { month, and } 120 \mathrm{~min} / \text { day, } 1 \text { day/week for } \\
\text { the second month, then } 160 \mathrm{~min} / \mathrm{month} \text {, } \\
36 \mathrm{months}\end{array}$ & $\begin{array}{c}\text { Global cognitive } \\
\text { function/Composite } \\
\text { score } \\
\text { Memory/FCSRT } \\
\text { Executive function/ } \\
\text { TMT-B } \\
\text { Information processing } \\
\text { speed/DSST } \\
\text { Verbal fluency/COWAT } \\
\text { Attention/Ten MMSE } \\
\text { orientation items } \\
\text { Depression/GDS } \\
\text { Mentality/ } \\
\text { Consequences of } \\
\text { everyday life } \\
\text { Subjective memory } \\
\text { function/Memory } \\
\text { functioning } \\
\text { Physical fitness/SPPB }\end{array}$ \\
\hline $\begin{array}{l}\text { Zuniga et al. } 2016 \\
\text { [56] }\end{array}$ & Т $66.4 \pm 5.7$ & $\begin{array}{c}179 \\
(62 / 117)\end{array}$ & $\begin{array}{l}\text { T: Physical activity } \\
\text { C: Active Control }\end{array}$ & $\begin{array}{c}60 \mathrm{~min} / \text { day, } 3 \text { days/week, } 12 \text { months } \\
50-75 \% \text { HRR }\end{array}$ & $\begin{array}{c}\text { Depression/PSS } \\
\text { Mentality/MUNSH } \\
\text { Subjective memory } \\
\text { function/FOF } \\
\text { Physical fitness/CHIPS }\end{array}$ \\
\hline
\end{tabular}


TABLE 1: Continued.

\begin{tabular}{|c|c|c|c|c|c|}
\hline Author/Year & Mean age & $\mathrm{N}(\mathrm{M} / \mathrm{F})$ & Intervention & Frequency, duration and intensity & Outcomes/measure \\
\hline Cox et al. 2013 [57] & $\begin{array}{l}\text { T: } 68.2 \pm 9.8 \\
\text { C: } 65.6 \pm 4.7\end{array}$ & $\begin{array}{c}170 \\
(84 / 86)\end{array}$ & $\begin{array}{l}\text { T: Combined } \\
\text { C: Control }\end{array}$ & $\begin{array}{c}50 \mathrm{~min} / \text { day, } 3 \text { days/week, } 6 \text { months } \\
\text { Moderate intensity }\end{array}$ & Mentality/SCI \\
\hline $\begin{array}{l}\text { Small et al. } 2006 \\
\text { [58] }\end{array}$ & $\begin{array}{l}\text { T: } 54 \pm 12 \\
\text { C: } 53 \pm 10\end{array}$ & $17(6 / 11)$ & $\begin{array}{c}\text { T: Physical activity } \\
\text { combined cognitive } \\
\text { training } \\
\text { C: Control }\end{array}$ & 30-45 $\mathrm{min} /$ day, 7 days/week, 2 weeks & $\begin{array}{c}\text { Memory/VLM } \\
\text { Verbal fluency/Word- } \\
\text { generation (letter- } \\
\text { fluency) test } \\
\text { Subjective memory } \\
\text { function/MFQ }\end{array}$ \\
\hline Barnes 2013 [59] & $\begin{array}{l}\text { T: } 74.8 \pm 6.1 \\
\text { C: } 73.9 \pm 6.3\end{array}$ & $95(34 / 61)$ & $\begin{array}{l}\text { MA-I/EX-I: Physical } \\
\text { activity combined } \\
\text { cognitive activity } \\
\text { MA-C/EX-I: Physical } \\
\text { activity } \\
\text { C: Active control }\end{array}$ & $\begin{array}{c}60 \mathrm{~min} / \text { day, } 3 \text { days/week, } 6 \text { months } \\
65-85 \% \text { of maximum HR }\end{array}$ & $\begin{array}{c}\text { Global cognitive } \\
\text { function/Composite } \\
\text { score } \\
\text { Memory/RAVLT } \\
\text { Executive function/ } \\
\text { TMT-B } \\
\text { Information processing } \\
\text { speed/DSST } \\
\text { Verbal fluency/Letter } \\
\text { and Category } \\
\text { Attention/UFVO }\end{array}$ \\
\hline $\begin{array}{l}\text { Nagamastsu et al. } \\
2013[60]\end{array}$ & $\begin{array}{l}\text { T: } 75.6 \pm 3.6 \\
\text { C: } 75.1 \pm 3.6\end{array}$ & 58 & $\begin{array}{l}\text { T: Physical activity } \\
\text { C: Active control }\end{array}$ & $\begin{array}{c}60 \mathrm{~min} / \text { day, } 2 \text { days/week, } 6 \text { months } \\
40-80 \% \mathrm{HRR}\end{array}$ & $\begin{array}{l}\text { Verbal Memory and } \\
\text { Learning/RAVLT }\end{array}$ \\
\hline
\end{tabular}

CNS, computerized CNS Vital Signs Memory Protocol test ; HKLLT, Hong Kong list learning test; WMS-III-VR, visuospatial reproduction subtest of the Wechsler Memory Scale III; CDR, Clinical Dementia Rating; ADAS-Cog, Alzheimer Disease Assessment Scale-Cognitive Subscale; DSC, digit symbol coding; VFT, verbal fluency total; BDI, Beck Depression Inventory; MCS, Medical Outcomes 36-Item Short Form (SF-36) mental component summary; PCS, SF-36 physical component summary; WMS, Wechsler Memory Scale; SQLP, the Subjective Quality of Life Profile; FCSRT, Free and Cued Selective Reminding Test; TMT-B, Trail Making Test B; DSST, Digit Symbol Substitution Test; COWAT, Controlled Oral Word Association Test; MMSE, Mini-Mental State Examination; GDS, the Geriatric Depression Scale; SPPB, the Short Physical Performance Battery; PSS, Perceived Stress Scale; MUNSH, Memorial University of New Foundland Scale of Happiness; FOF, Frequency of Forgetting scale; CHIPS, Cohen-Hoberman Inventory of Physical Symptoms; SCI, the Stage of Change Instrument; VLM, verbal learning and memory test; MFQ, The Memory Functioning Questionnaire; RAVLT, Rey Auditory Verbal Learning Test; UFVO, Useful Field of View.

exclusion criteria for their participants. The interventions of the included studies were physical activity combined with cognitive training. Multiple-modality exercise with mindmotor training (M4) [47], supervised strategy-based memory training sequentially after stationary cycling (SEQ) [51], Dejian Mind-Body intervention (DMBI) [52], and physical activity and cognitive stimulation [55] were also practiced. The majority of interventions occurred in physical activity combined with cognitive training, except for four studies reporting the physical activity.

The frequency of intervention varied from 1 to 5 sessions weekly and 30-150 mins per session. The duration of the intervention was from 2 weeks to 36 months. Of these, 11 studies compared combined physical-cognitive interventions with stretching and/or tone exercise, cognitive training, social activities, or health education. Since the lower intensity of those activities did not significantly alter the exercise habits of the participants, we believed that those activities did not differ. A heart rate reserve $[51,56,60]$ from $40 \%$ progressed to $80 \% ; 60-85 \%$ of maximum heart rate $[47,54,59]$ or "moderate intensity" $[53,57]$ was applied to control the intensity of physical activity in the included studies; there was no description in others $[52,55,58]$. The intervention in the control group was the very low intensity of activity/exercise such as social activities, stretching, balance and tone exercise, or health education.

The meta-analysis was conducted to analyze the cognitive outcomes and self-report outcomes, including cognitive function, psychological well-being, and emotion. There was a wide variety of measurement tools applied to evaluate the same cognitive domain within a study or among studies, including MMSE, ADAS-Cog, CDR, Logical Memory subtest of the Wechsler Memory Scale-Revised (WMS-LM), digit symbol cording (DSC), Trail Making Test (Parts A and B), Stroop test, letter verbal fluency test (LVFT), and category verbal fluency test (CVFT). Otherwise, there were kinds of scales to evaluate the mentality and physical fitness (i.e., The Short Form-12, SF-36), depression (i.e., BDI, 15-item Geriatric Depression Scale), and subjective memory impairment (i.e., Frequency of Forgetting Scale, Memory Performance).

3.3. Risk of Bias of Included Studies. The quality of the RCTs included was assessed by two reviewers independently by using the RoB 2 tool, a revised Cochrane RoB tool for randomized trials [56]. The risk of bias in each study is illustrated in Figure 2. 


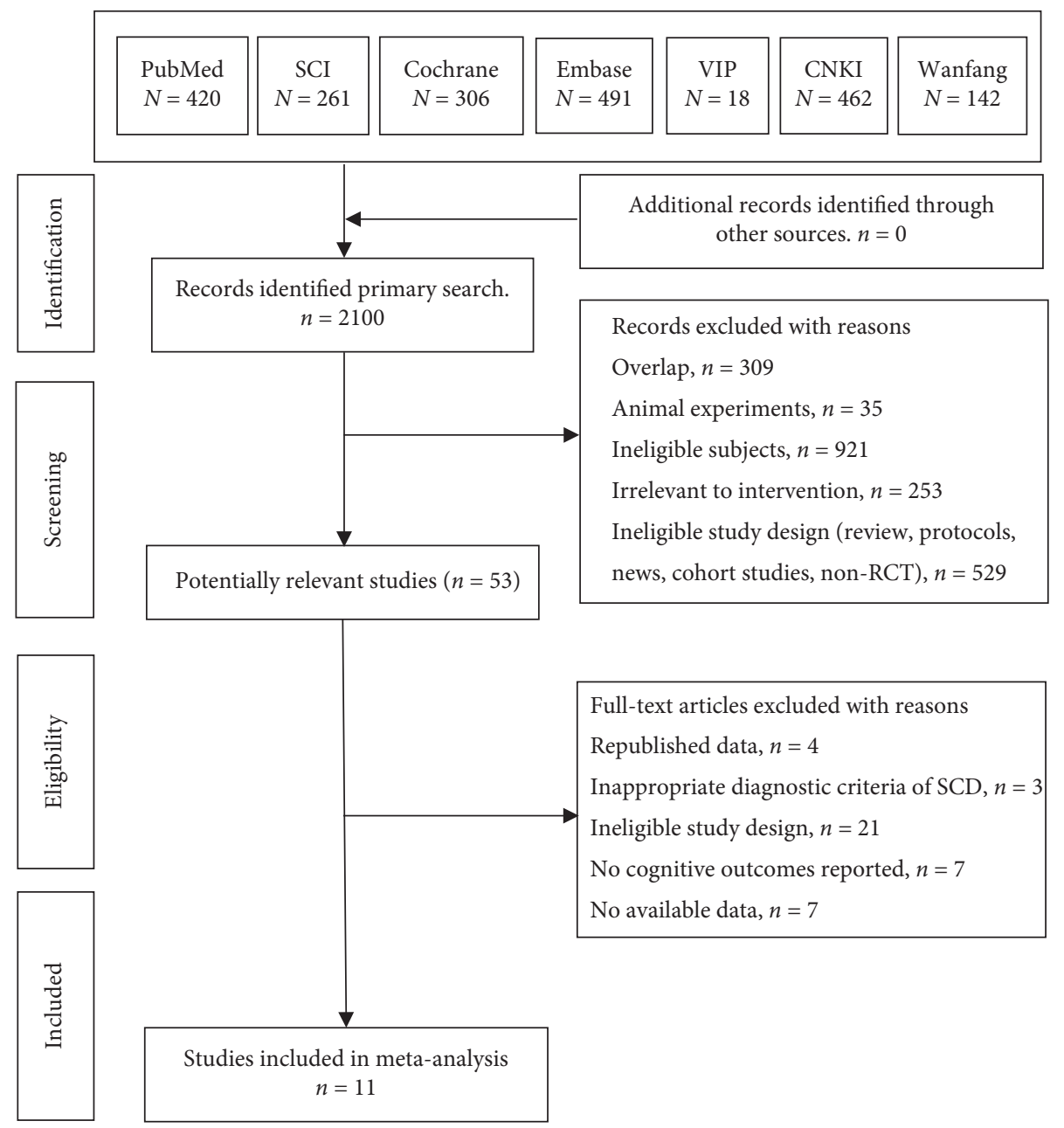

FIgURE 1: The literature searches and select flow diagram of the included studies. SCI, science citation index; VIP, Chinese Science and Technology Periodical database; CNKI, China National Knowledge Infrastructure; SCD, subjective cognitive decline; RCT, randomized controlled trial.

Five studies $[47,53,55,57,60]$ were identified as having low risk in the randomization process, and six studies [51, $52,54,56,58,59]$ were identified as having uncertain risk. The risk of deviations from intended interventions was low in six studies $[47,53,55,57,59,60]$ and uncertain in five studies [51, 52, 54, 56, 58]. All studies [47, 51-60] had a low risk for missing outcome data. Seven studies $[47,53-55,57$, $59,60]$ were identified as having low risk and four studies $[51,52,56,58]$ were identified as having high risk related to measurement of the outcome. Furthermore, seven studies $[51,53,56-60]$ were uncertain risk and one study [52] was high risk in terms of the reported result selection. Finally, the risk of overall bias was noted as low in two studies $[53,55]$, uncertain in five studies $[47,54,57,59,60]$, and high $[51,52$, $56,58]$ in four studies.

\subsection{Effects of Interventions}

3.4.1. Memory Self-Efficacy (MSE). Two studies [55, 58] involving 787 participants compared the combined cognitive-physical interventions with control groups in terms of subjective memory function measured by memory functioning [55] and the Memory Functioning Questionnaire (MFQ) [58]. Compared with the control group, no significant improvement was found in the combined physicalcognitive interventions group in the subjective memory function $(n=787, \mathrm{SMD}=0.04,95 \%$ CI -0.10 to 0.18 , $P=0.54$; Figure 3).

3.4.2. Objective Cognitive Function. Seven studies $[55,59]$ reported the effects of combined cognitive-physical interventions on objective cognitive function in participants with SCD including global cognitive function, memory, executive ability, attention, and verbal fluency.

Three studies reported the effects of combined physicalcognitive interventions on composite memory, which were measured by the Free and Cued Selective Reminding Test, WMS, Verbal, and Visual Memory Test [51, 54, 55]. Two studies $[47,52]$ reported the effects of combined physicalcognitive interventions on immediate memory ability which was measured by Spatial Span and WMS. Three studies $[51,55,59]$ involving a number of 889 participants 

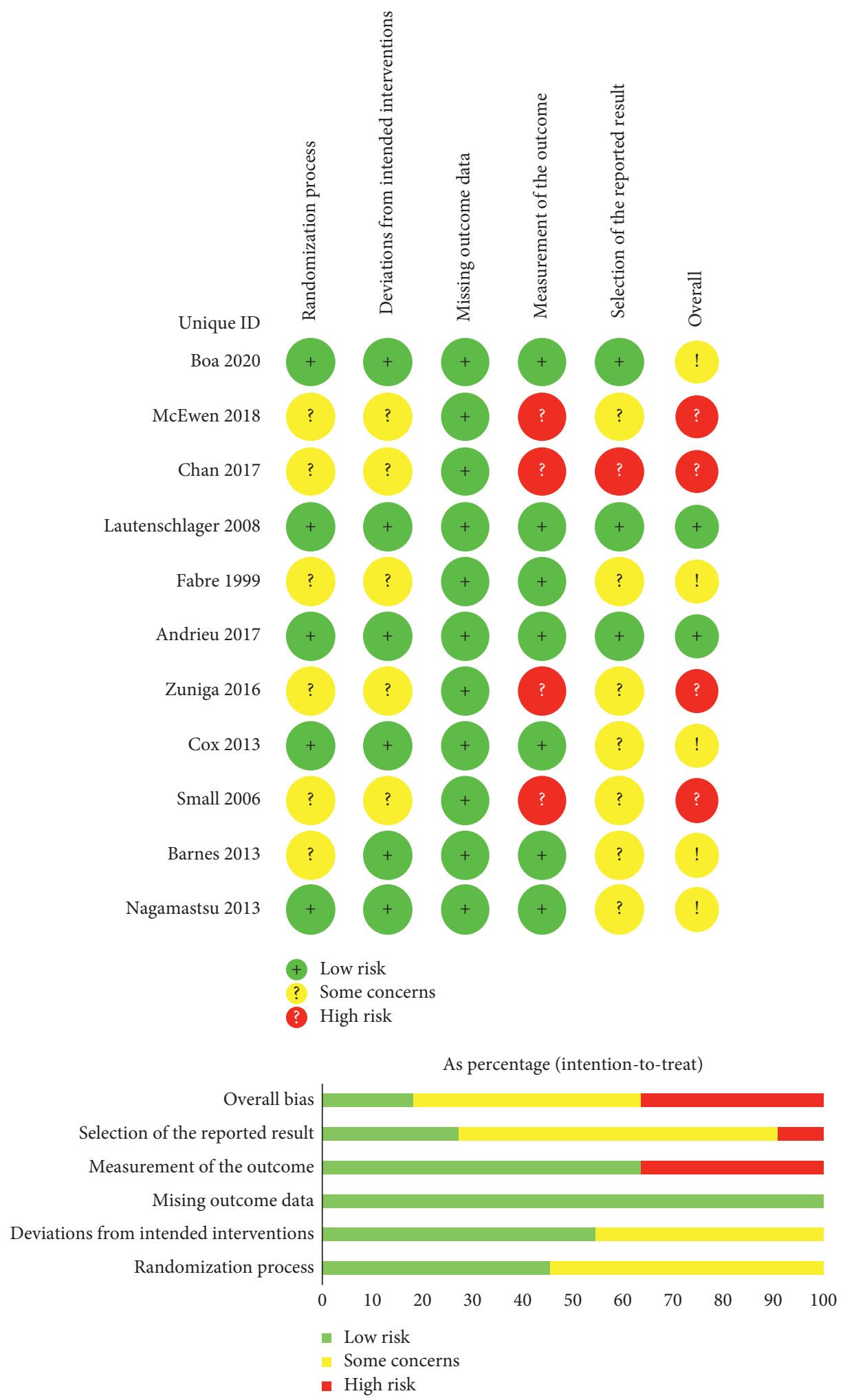

FIGURE 2: Summary of the risk of bias of the included studies: review authors' judgments about each risk of bias item for each included study.

compared the combined physical-cognitive interventions and control groups in terms of attention, which was measured by Stroop test [51], ten MMSE orientation items [55], and Useful Field of View (UFOV) [59]. The effects of combined physical-cognitive interventions on executive ability were evaluated in three studies $[51,55,59]$ with a total of 882 participants using the Trail Making Test part B $[55,59]$ or CNS [51]. Three studies with 851 participants assessed verbal fluency using the COWAT [55], wordgeneration (letter-fluency) test [58], or verbal fluency test 


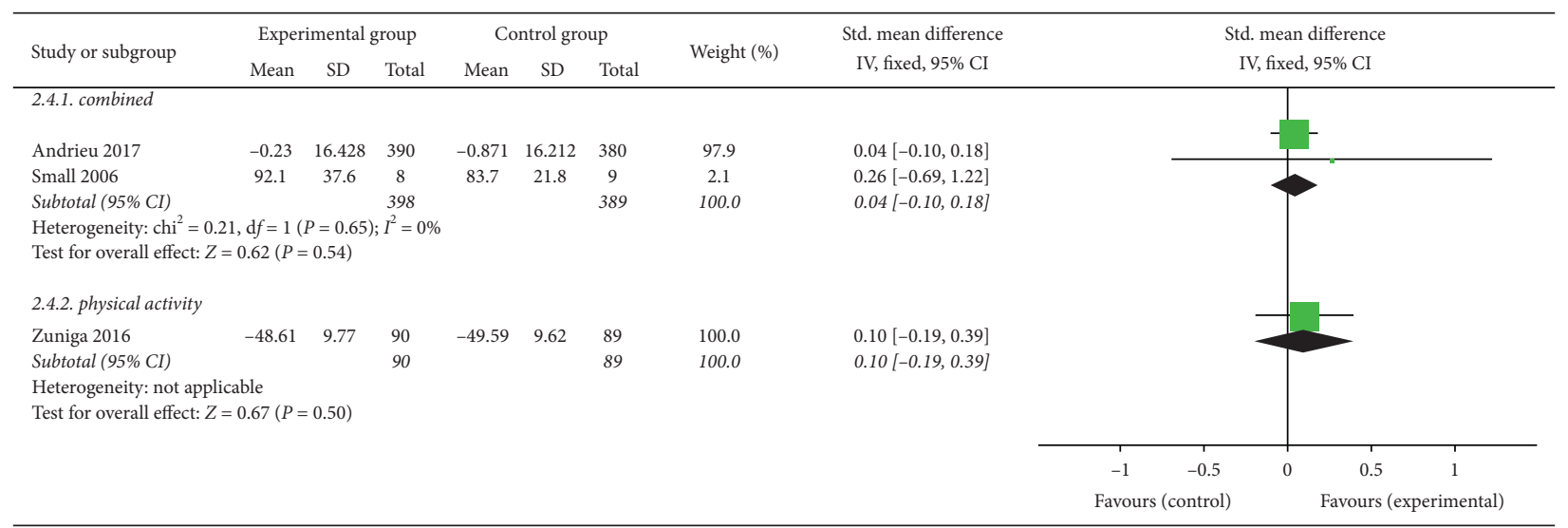

Figure 3: Forest plot for combined physical-cognitive interventions or physical activity on MSE. Combined: combined physical-cognitive interventions.

[59]. Owing to the different tools, we used SMD scores. The results showed that participants in the combined physicalcognitive interventions group had a significant improvement in SMD scores of objective cognitive function compared with the controls $(n=5679, \mathrm{SMD}=0.12,95 \%$ CI 0.07 to $0.17, P<0.001 ; I^{2}=0 \%$, the fixed-effect model; Figure 4 ). Moderator analyses were not conducted because of the heterogeneity statistics for the fixed-effect model confirming that there was no heterogeneity $\left(I^{2}=0 \%\right)$.

The effects of physical activity on objective cognitive function were evaluated in four studies [53, 54, 59, 60]. Meta-analysis showed that physical activity had a significant improvement in SMD scores of objective cognitive function compared with controls $(n=1328$, $\mathrm{SMD}=0.13,95 \%$ CI 0.03 to $0.24, P=0.02 ; I^{2}=0 \%$, the fixed-effect model; Figure 5).

3.4.3. Psychological Well-Being. Two studies $[54,55]$ reported the effects of combined physical-cognitive interventions on mentality, which was measured by SQLP [54], and the consequences of everyday life [55]. The results showed that participants in the combined physical-cognitive interventions group had no significant improvement in SMD scores compared with controls $(n=786, \mathrm{SMD}=0.12$, $95 \% \mathrm{CI}-0.02$ to $0.26, P=0.09$; Figure 6 ). Four studies $[53,54,56,57]$ involving 535 participants assessed the effects of physical activity on mentality measured by SF-36 mentality component summary [53], SQLP [54], Memorial University of Newfoundland Scale of Happiness [56], and the Stage of Change Instrument [57]. Meta-analysis showed physical activity had significant improvement of mentality ( $n=535, \mathrm{SMD}=0.22,95 \%$ CI 0.05 to $0.39, P=0.01, I^{2}=22 \%$, the fixed-effect model; Figure 6).

3.4.4. Emotion (Depression). Three studies with 851 participants assessed depression using the Subjective Quality of Life Profile (SQLP) [54], Geriatric Dementia Scale (GDS) [55], and Perceived Stress Scale (PSS) [56]. Meta-analysis showed that combined physical-cognitive interventions had no significant improvement in depression $(n=965$,
SMD $=-0.09,95 \%$ CI -0.21 to $0.04, P=0.18$; Figure 7 ). Compared to the usual care control group, there was no significant improvement in the Beck Depression Inventory (BDI) scores in the physical activity group in two studies involving 186 participants $(n=186, \mathrm{SMD}=-0.11,95 \% \mathrm{CI}$ -0.39 to $0.18, P=0.47$; Figure 7 ).

3.4.5. Safety and Adverse Events. No serious adverse events were reported during combined physical-cognitive interventions in the included studies. One study [53] reported one participant with foot pain and gout intervention, and another study [60] reported two people with shortness of breath and four falls; however, they were without any physical injury.

\section{Discussion}

4.1. Some Findings. In this present review, we explore the effect of combined physical-cognitive interventions on MSE, objective cognitive ability, psychological well-being, and emotion in comparison with the usual lifestyle in the elderly with SCD. Eleven eligible studies were included, and pooled analyses were not performed, due to different assessment scales and number of participants, kinds of activities, and methodological quality of the included studies. The results indicated a positive association in the elderly with SCD intervention trials to date, including objective cognitive function and well-being, combined physical-cognitive interventions, or only physical activity intervention, and they were added to the qualitative review because only RCTs were included.

Regarding objective cognitive function, the pooled results showed that combined physical-cognitive interventions yielded significant improvements in SCD elderly in our meta-analysis. We found that combined cognitive-physical interventions conferred a significant benefit on attention, which is partly consistent with other studies. A review has shown the beneficial effects of combined cognitive and exercise training for improving cognitive functions and functional status in older adults with and without cognitive impairment. In line with this, a meta-analysis revealed 


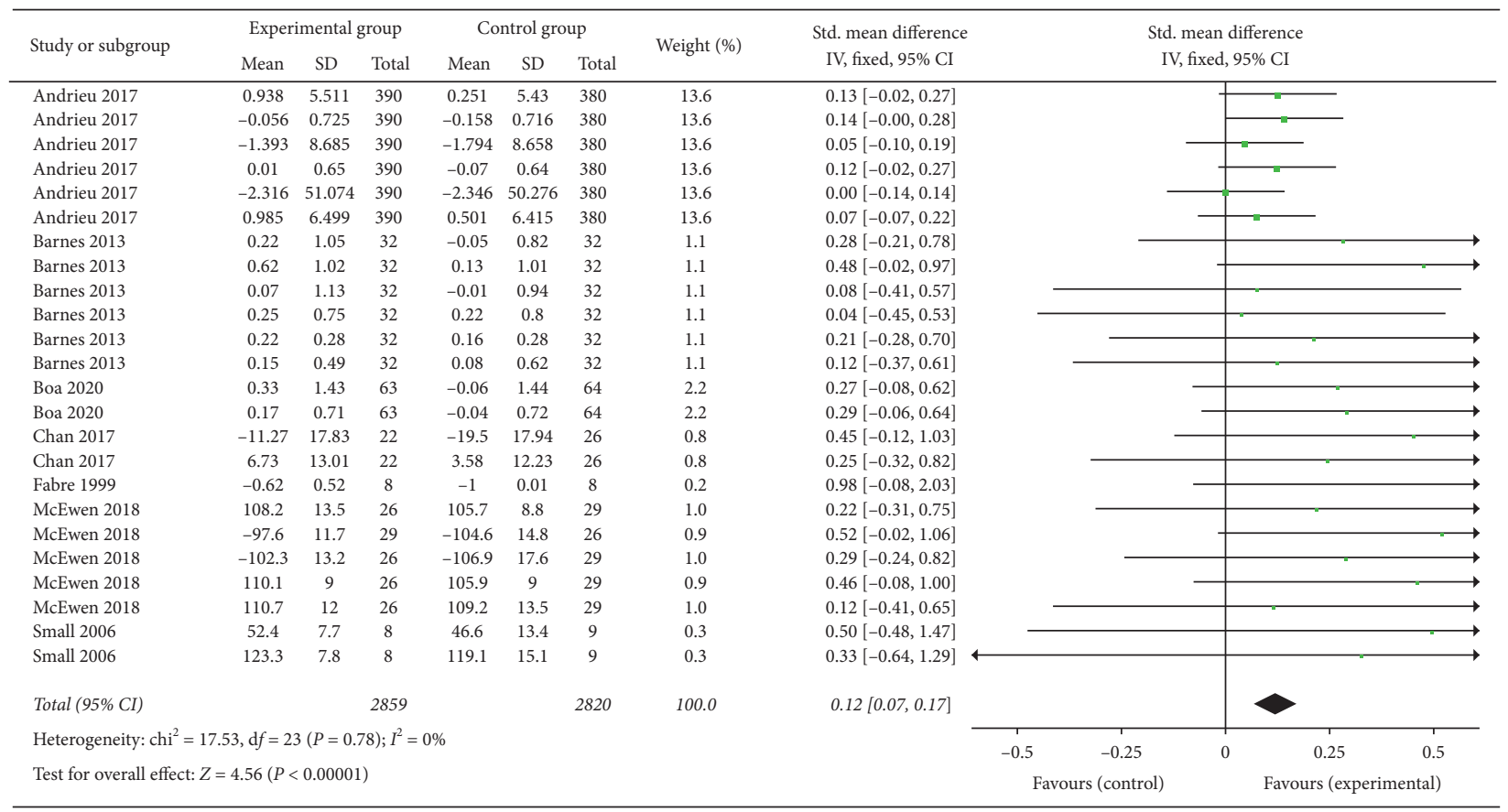

FIGURE 4: Forest plot for combined physical-cognitive interventions on objective cognitive function.

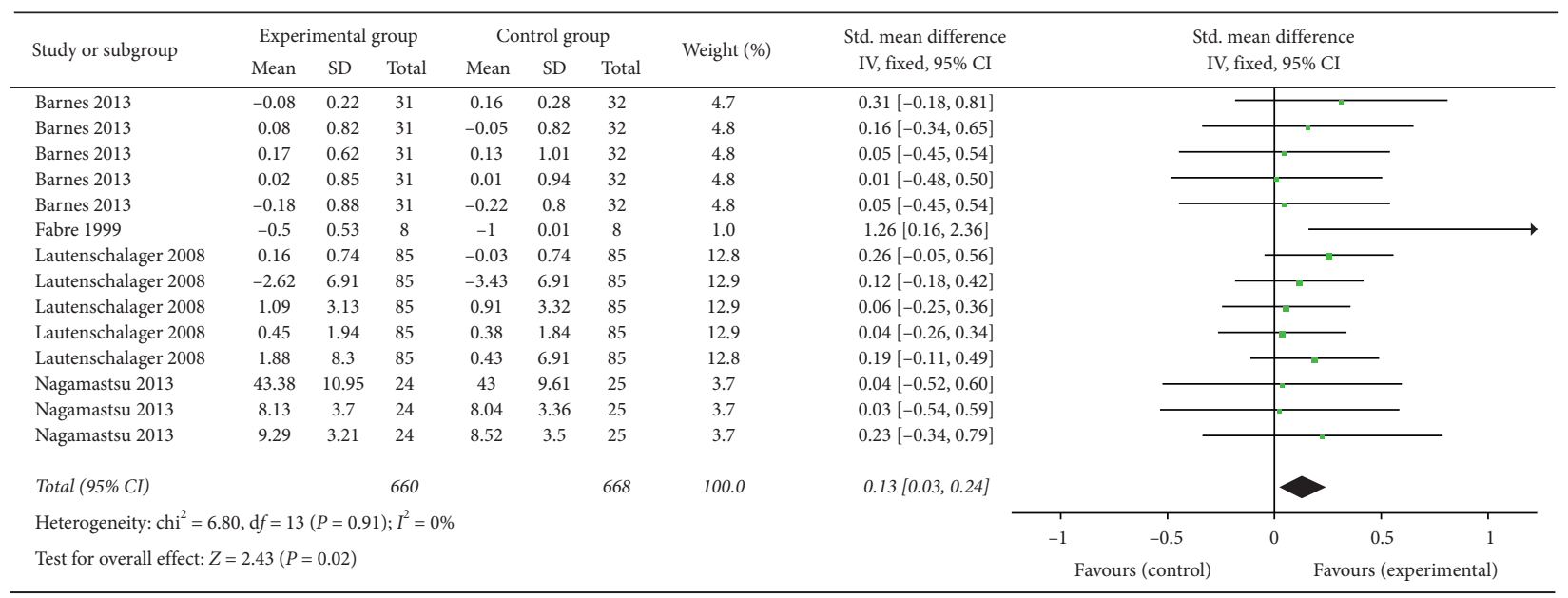

FIGURE 5: Forest plot for physical activity on objective cognitive function.

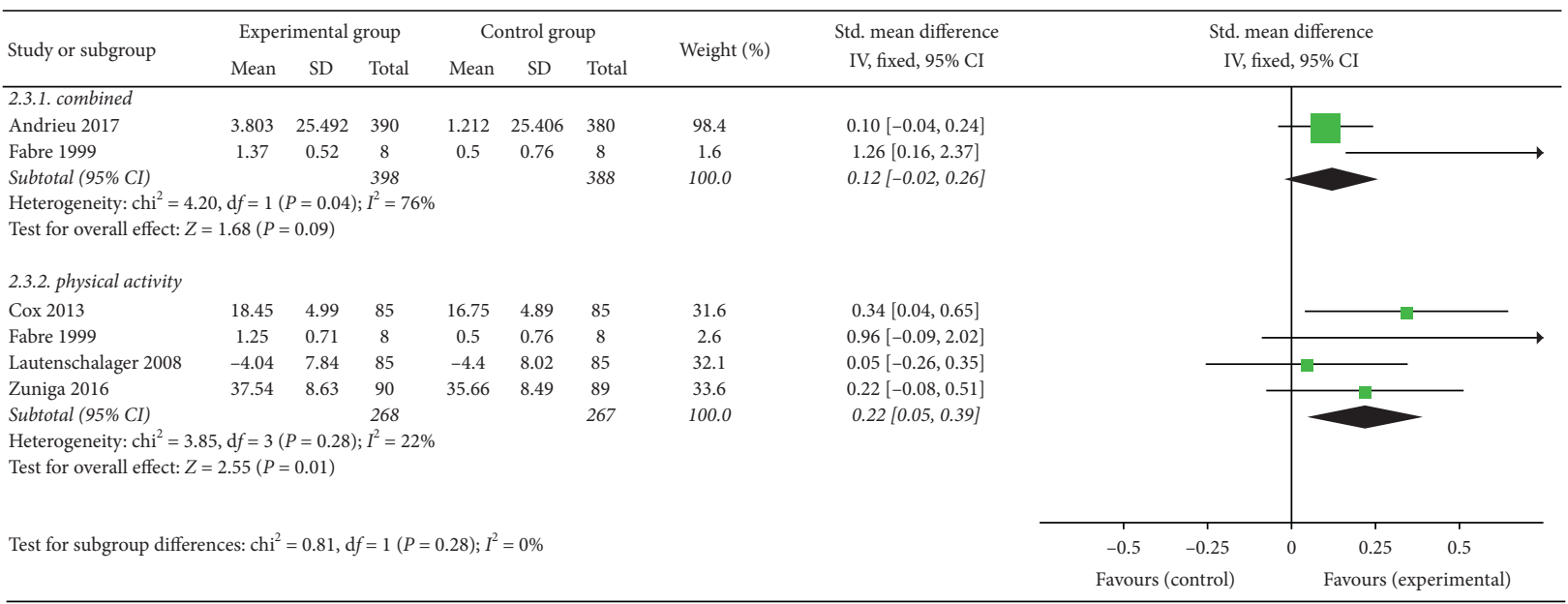

FIGURE 6: Forest plot for combined physical-cognitive interventions or physical activity on psychological well-being. Combined: combined physical-cognitive interventions. 


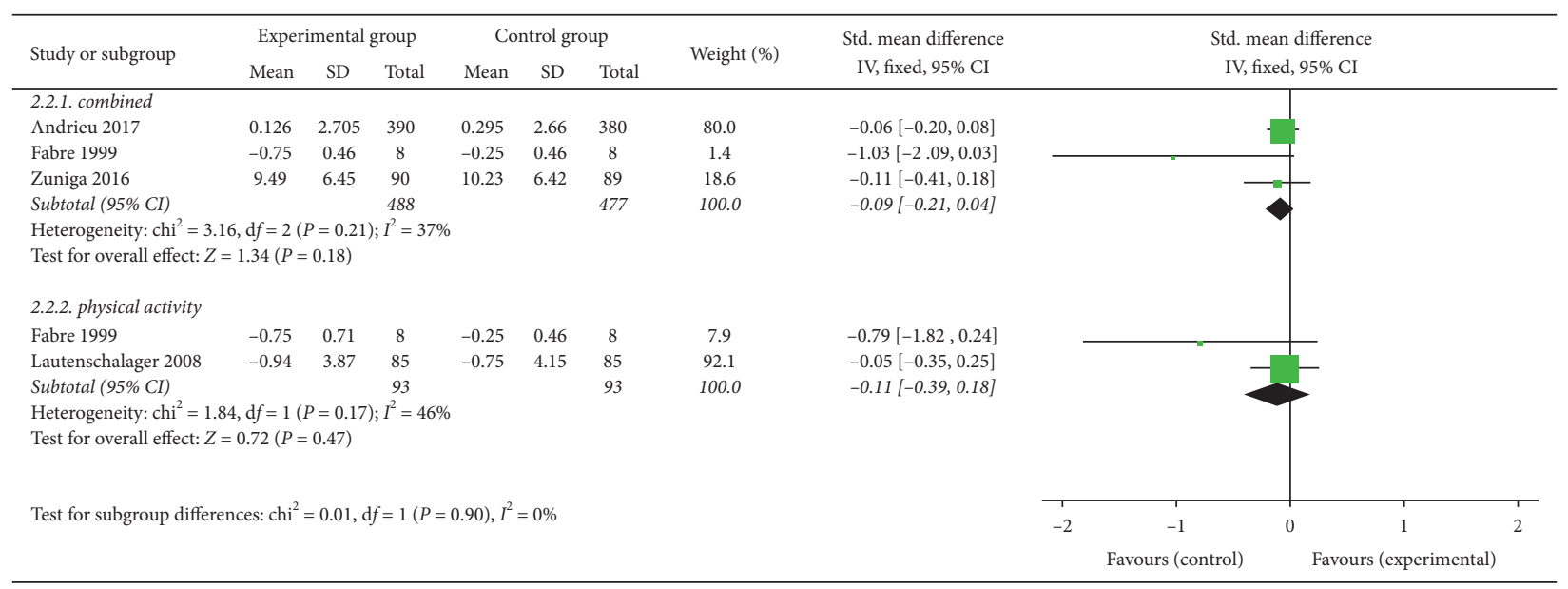

FIGURE 7: Forest plot for combined physical-cognitive interventions or physical activity on depression. Combined: combined physicalcognitive interventions.

significantly larger effects of combined cognitive and physical interventions, compared with both single exercise training and a control group on attention in healthy older adults [63]. McEwen's study showed that the SEQ group has a significantly increased reaction time while single stationary cycling training showed no significant changes in 55 older adults with subjective memory impairment [51].

In addition, we found a positive effect of combined physical-cognitive interventions on verbal fluency; this finding was consistent with the results of Small and colleagues which reported that a 14-day healthy longevity lifestyle program objectively demonstrated greater verbal fluency scores in older individuals with mild self-reported memory complaints but normal baseline memory performance scores [58].

In the present study, our results showed that the combined interventions are effective in improving objective cognitive function in subjective cognitive decline (SCD) older adults. As we know, SCD is identified as a condition where some individuals experience subjective cognitive complaints, but the objective examination did not show signs of cognitive decline. For the patients with SCD, the decline in cognitive function is relatively slight; therefore, the significant improvement with large SMD after the intervention may be not easy. For example, a systematic review and meta-analysis suggested that cognitive training interventions led to a small, statistically significant improvement in objective cognitive performance for SCD $(g=0.13,95 \%$ CI 0.01 to $0.25 ; P=0.03$ ).

However, SCD is considered as the state between normal aging and (MCI) and may be the preclinical stage of (AD), with high risks of conversion to MCI or AD [52]. SCD may be a key window treatment period to delay the deterioration of cognitive function; early intervention and rehabilitation can help the reversal of cognitive decline [52]. Therefore, the effective difference may show the potential value of combined physical-cognitive interventions in improving objective cognitive ability and preventing the conversion of SCD to MCI or AD. However, due to the small SMD, the clinical importance may be not clear; further studies will be needed to explore the potential benefits of this therapy in preventing the conversion of SCD to MCI or AD.

Previous studies suggested that a cognitively challenging environment may enhance the neural and cognitive benefits that are elicited by physical activity $[63,64]$. Animal studies showed that combined physical activity and environmental enrichment induce a significant enhancement in dendritic arborization of CA3 hippocampal neurons than either physical activity or an enriched environment, each on its own [41]. We consider that simultaneous physical exercise and cognitive training might increase the impact on neuroplasticity based on human and animal research, which has found that combined physical exercise and cognitive training yielded greater increases in new neurons in mice than either intervention alone $[41,43,44]$. Further study will be needed to verify the results.

Whether combined physical-cognitive interventions could improve memory ability is a controversial issue. Several studies have shown that combined physical-cognitive interventions have improved memory function, including composite memory, recent memory, and delayed memory $[52-54,59,60]$. Conversely, the remaining studies included showed combined physicalcognitive interventions to have negative results in memory ability $[47,51,54,65]$. The changes in executive functioning differed significantly compared with the control group in only one study [52]. A meta-analysis of controlled trials with older adults presented a potential advantage at improving executive functions by physical and cognitive interventions. We speculate that there were several reasons. Firstly, the mode of combination, intervention frequency, intervention length, session length, and setting also were considered as potential moderators; secondly, the small sample size and lack of monitoring of the compliance of participants prevent any firm conclusions from being drawn; furthermore, the measures of cognition used varied across the studies that included cognitive tests, limiting its comparability. Further studies will be needed to explore the effects of combined physicalcognitive interventions on global cognitive function and 
the specific domains of cognition, including memory ability and executive ability.

Self-report memory complaints may predict the potential increased risk of dementia in objective cognitive performance. The English Longitudinal Study of Aging found that aged 50 years and above had a negative correlation between subjective memory complaints and objective performance in all cognitive domains [8]. In Zuniga's study, a significant main effect of subjective memory on measures of well-being indicated that individuals with the fewest memory complaints were significantly happier and lower perceived stress/physical symptoms reported across all measurement occasions [56]. However, our meta-analysis containing three studies did not show significant improvement in MSE in the combined physical-cognitive interventions $[55,56,58]$. To consider that the small sample size and the MSE were not a primary outcome for any of the combined physical-cognitive interventions studies, in these studies, further work is required to explore the role of combined physical-cognitive interventions in improving MSE.

In other cases, SCD may be associated with emotion, personality, and physical health concerns. Our findings indicated that there was a statistically significant improvement in well-being with only physical activity intervention. The present findings are comparable with a previously published meta-analysis, which conferred a small but significant benefit on psychological well-being after cognitive training interventions in older adults with SCD [66]. In Zuniga's study, a significant main effect of subjective memory on measures of well-being indicated that individuals with the fewest memory complaints were significantly happier and lower perceived stress/physical symptoms reported across all measurement occasions [56]. Significantly high importance was attributed to changes in self-efficacy in home-based physical activity programmers with older adults with memory complaints [57]. However, it is important to note that there is sometimes an unproven assumption that physical activity enhances a patient's overall well-being by preserving cognitive function [53]. The ground on these pieces of evidence, the well-being of supports that suggests cognitive impairment may have a negative impact on quality of life indicators in SCD elderly.

In the present study, we found that the combined interventions are effective in improving objective cognitive function in SCD older adults which may show the potential value of combined physical-cognitive interventions in improving objective cognitive ability and preventing the conversion of SCD to MCI or AD. However, due to the small SMD, the clinical importance may be not clear. Further studies will be needed to explore the potential benefits of this therapy in preventing the conversion of SCD to MCI or AD.

4.2. Strengths and Limitations. The strengths of this review belong to its systematic approach in which methodologically stronger study designs were used. First, we focused on types of combined physical activity and cognitive training, rather than nonpharmacologic interventions or all interventions. Furthermore, only RCTs were included in this meta-analysis. In addition, to reduce potential confounding bias, we only investigated older adults with SCD.

There are several limitations in the present study. First, the included studies with different levels of quality demonstrated methodologically heterogeneity that may have impacted our interpretation of the results. Second, the heterogeneity of intervention characteristics in the included studies should be considered, including the type and time, frequency, and duration. The type and time of combined physical-cognitive interventions used in the included studies were physical activity (e.g., walking, Dejian mind-body intervention, aerobic cycling, stretching, and jogging) and cognitive training (e.g., memory training, cognitive stimulation), which varied from 45 to $150 \mathrm{~min}$ per session. The intervention duration of the included studies ranged from 2 weeks to 3 years. Studies have investigated the decay after intervention cessation was less. Therefore, the optimal intervention design for the intervention effects remains unclear; more research is warranted. Third, a variety of noncompatible measurement tools were used in the included studies. Future studies should use more objective testing methods or instruments (e.g., functional MRI, event-related potential, and PET). Fourth, few studies (only two studies) reported the comparison between the combined interventions and cognitive or physical activity alone on SCD according to our current knowledge (according to the selection/identification/inclusion criteria of studies in the present study). In addition, there are no common outcomes in the two studies above; therefore, the meta-analysis of the combined interventions as compared to cognitive or physical intervention alone does not include common outcomes in the main findings. We will continue to pay attention to this issue in the future. Furthermore, most included studies were small samples. Last but not least, it is impossible to blind participants in a physical activity intervention trial; therefore, performance bias may be inevitable.

4.3. Clinical Implications and Recommendations for Future Studies. The combined interventions are effective in improving objective cognitive function in SCD older adults which may show the potential value of combined physicalcognitive interventions in improving objective cognitive ability and preventing the conversion of SCD to MCI or AD and no adverse effects. We provide clinicians with positive evidence of an effective option to recommend those older adults with SCD who would benefit from increasing combined physical-cognitive training.

Future studies in this field should use appropriate exercise modes, exercise prescriptions including training intensity, and frequency, such as the exercise training principles suggested by Bherer [63] or the global recommendations on physical activity for health provided by the WHO. In addition, if possible, the duration of training should be at least 6 months or longer [35]. Furthermore, more sensitive and objective measurement tools should be used. Finally, authors should follow the CONSORT guidelines when reporting their studies to allow better evaluation of the quality. 


\section{Conclusion}

The combined interventions are effective in improving objective cognitive function in SCD older adults which may show the potential value of combined physical-cognitive interventions in improving objective cognitive ability and preventing the conversion of SCD to MCI or AD. However, considering the quality of included studies, discrepancies in the types, frequencies, and durations of interventions, as well as the limitations of the included studies, the findings must be interpreted cautiously. To draw specific and accurate conclusions regarding the potential enhancement effects of combined physical activity and cognitive training interventions on the objective cognitive ability, well-being, and MSE of SCD elderly, more rigorously designed and standardized training protocols of large-scale RCTs are required in future research.

\section{Data Availability}

All data analyzed during this study are included in this article. The datasets used and/or analyzed during the current study are available from the corresponding author on reasonable request.

\section{Additional Points}

Search Strategy. \#1 (((((“subjective cognitive impairment”) OR "cognitive complaints") OR "subjective cognitive decline") OR "memory complaints") OR "subjective memory impairment") OR "Subjective memory decline". \#2 ((“"intervention”) OR “training”) OR “exercise”) OR “activity”. \#3 \#1AND\#2.

\section{Disclosure}

The funders have no role in the study design, data collection and analysis, decision to publish, or preparation of the manuscript.

\section{Conflicts of Interest}

The authors have no conflicts of interest to report.

\section{Authors' Contributions}

LJ conceived and designed the study and wrote and revised the manuscript; SQQ, XSR, GS, and YY were the research assistants who helped with data search, extraction, methodological assessment, and other aspects of the study; XR revised the manuscript; SQQ and XSR contributed equally to this study. All authors contributed to the writing of the manuscript and have read and approved the final manuscript version for publication.

\section{Acknowledgments}

This study was supported by the National Natural Science Foundation of China (Grant no. 81904270), Natural Science
Foundation of Fujian Province (Grant no. 2019J01362), and Educational Department of Fujian Province Outstanding Youth Scientific Research Talent Cultivation Program (Grant no. MinJiaoKe [2018]47).

\section{References}

[1] Alzheimer's Association, “2020 Alzheimer's disease facts and figures,” Alzheimers Dement, vol. 16, pp. 391-460, 2020.

[2] H. Brody, R. Hodson, and E. Batty, "Alzheimer's disease," Nature, vol. 559, p. S1, 2018.

[3] P. L. Valenzuela, A. Castillo-García, J. S. Morales et al., "Exercise benefits on Alzheimer's disease: State-of-the-science," Ageing Research Reviews, vol. 62, Article ID 101108, 2020.

[4] Y. Lin, P.-Y. Shan, W.-J. Jiang, C. Sheng, and L. Ma, "Subjective cognitive decline: preclinical manifestation of Alzheimer's disease," Neurological Sciences, vol. 40, no. 1, pp. 41-49, 2019.

[5] I. Lazarou, S. Nikolopoulos, S. I. Dimitriadis, I. Kompatsiaris, M. Spilioti, and M. Tsolaki, "Is brain connectome research the future frontier for subjective cognitive decline? A systematic review," Clinical Neurophysiology, vol. 130, no. 10, pp. 1762-1780, 2019.

[6] E. Chipi, N. Salvadori, L. Farotti, and L. Parnetti, "Biomarkerbased signature of Alzheimer's disease in pre-MCI individuals," Brain Sciences, vol. 9, no. 9, p. 213, 2019.

[7] F. Jessen, R. E. Amariglio, M. van Boxtel et al., "A conceptual framework for research on subjective cognitive decline in preclinical Alzheimer's disease," Alzheimer's \& Dementia, vol. 10, no. 6, pp. 844-852, 2014.

[8] G. Chen, K. Yang, W. Du, X. Hu, and Y. Han, "Clinical characteristics in subjective cognitive decline with and without worry: baseline investigation of the SILCODE study," Journal of Alzheimer's Disease, vol. 72, no. 2, pp. 443-454, 2019.

[9] F. Jessen, R. E. Amariglio, R. F. Buckley et al., "The characterisation of subjective cognitive decline," The Lancet Neurology, vol. 19, no. 3, pp. 271-278, 2020.

[10] A. J. Mitchell, H. Beaumont, D. Ferguson, M. Yadegarfar, and B. Stubbs, "Risk of dementia and mild cognitive impairment in older people with subjective memory complaints: metaanalysis," Acta Psychiatrica Scandinavica, vol. 130, no. 6, pp. 439-451, 2014.

[11] A. C. van Harten, M. M. Mielke, D. M. Swenson-Dravis et al., "Subjective cognitive decline and risk of MCI," Neurology, vol. 91, no. 4, pp. e300-e312, 2018.

[12] S. Verfaillie, T. Timmers, R. Slot et al., "Amyloid-beta load is related to worries, but not to severity of cognitive complaints in individuals with subjective cognitive decline: the science project," Front Aging Neurosci, vol. 11, p. 7, 2019.

[13] R. E. Amariglio, J. A. Becker, J. Carmasin et al., "Subjective cognitive complaints and amyloid burden in cognitively normal older individuals," Neuropsychologia, vol. 50, no. 12, pp. 2880-2886, 2012.

[14] R. F. Buckley, B. Hanseeuw, A. P. Schultz et al., "Regionspecific association of subjective cognitive decline with tauopathy independent of global $\beta$-amyloid burden," JAMA Neurology, vol. 74, no. 12, p. 1455, 2017.

[15] A. J. Saykin, H. A. Wishart, L. A. Rabin et al., "Older adults with cognitive complaints show brain atrophy similar to that of amnestic MCI," Neurology, vol. 67, no. 5, pp. 834-842, 2006. 
[16] L. Scheef, A. Spottke, M. Daerr et al., "Glucose metabolism, gray matter structure, and memory decline in subjective memory impairment," Neurology, vol. 79, no. 13, pp. 1332-1339, 2012.

[17] A. Perrotin, R. La Joie, V. de La Sayette et al., "Subjective cognitive decline in cognitively normal elders from the community or from a memory clinic: differential affective and imaging correlates," Alzheimer's \& Dementia, vol. 13, no. 5, pp. 550-560, 2017.

[18] P. Vannini, B. Hanseeuw, C. E. Munro et al., "Hippocampal hypometabolism in older adults with memory complaints and increased amyloid burden," Neurology, vol. 88, no. 18, pp. 1759-1767, 2017.

[19] S. L. Risacher, S. Kim, K. Nho et al., "APOE effect on Alzheimer's disease biomarkers in older adults with significant memory concern," Alzheimer's \& Dementia, vol. 11, no. 12, pp. 1417-1429, 2015.

[20] S. Moreno-Grau, O. Rodríguez-Gómez, Á. Sanabria et al., "Exploring APOE genotype effects on Alzheimer's disease risk and amyloid $\beta$ burden in individuals with subjective cognitive decline: the FundacioACE Healthy Brain Initiative (FACEHBI) study baseline results," Alzheimer's \& Dementia, vol. 14, no. 5, pp. 634-643, 2018.

[21] J. I. Ali, C. M. Smart, and J. R. Gawryluk, "Subjective cognitive decline and APOE $\varepsilon 4$ : a systematic review," Journal of Alzheimer's Disease, vol. 65, no. 1, pp. 303-320, 2018.

[22] G. Livingston, A. Sommerlad, V. Orgeta et al., "Dementia prevention, intervention, and care," The Lancet, vol. 390, no. 10113, pp. 2673-2734, 2017.

[23] S. M. Beck, H. Ruge, C. Schindler et al., "Effects of Ginkgo biloba extract EGb 761 on cognitive control functions, mental activity of the prefrontal cortex and stress reactivity in elderly adults with subjective memory impairment - a randomized double-blind placebo-controlled trial," Human Psychopharmacology: Clinical and Experimental, vol. 31, no. 3, pp. 227242, 2016.

[24] E. L. Boespflug, R. K. McNamara, J. C. Eliassen, M. D. Schidler, and R. Krikorian, "Fish oil supplementation increases event-related posterior cingulate activation in older adults with subjective memory impairment," The Journal of Nutrition, Health \& Aging, vol. 20, no. 2, pp. 161-169, 2016.

[25] M. R. H. Brautigam, F. A. Blommaert, G. Verleye, J. Castermans, E. N. H. Jansen Steur, and J. Kleijnen, "Treatment of age-related memory complaints with Ginkgo biloba extract: a randomized double blind placebo-controlled study," Phytomedicine, vol. 5, no. 6, pp. 425-434, 1998.

[26] J. Zhu, R. Shi, S. Chen et al., "The relieving effects of BrainPower advanced, a dietary supplement, in older adults with subjective memory complaints: a randomized, doubleblind, placebo-controlled trial," Evidence-Based Complementary and Alternative Medicine, vol. 2016, Article ID 7898093, 16 pages, 2016.

[27] B. Metternich, D. Kosch, L. Kriston, M. Härter, and M. Hüll, "The effects of nonpharmacological interventions on subjective memory complaints: a systematic review and metaanalysis," Psychotherapy and Psychosomatics, vol. 79, no. 1, pp. 6-19, 2010.

[28] C. M. Smart, N. A. Corson, J. E. Karr et al., "Non-pharmacologic interventions for older adults with subjective cognitive decline: systematic review, meta-analysis, and preliminary recommendations," Neuropsychology Review, vol. 27, no. 3, pp. 245-257, 2017.
[29] World Health Organization, Global Action Plan on Physical Activity 2018-2030, World Health Organization, Geneva, Switzerland, 2018.

[30] T. W. H. Chong, E. Curran, K. A. Ellis et al., "Physical activity for older Australians with mild cognitive impairment or subjective cognitive decline-a narrative review to support guideline development," Journal of Science and Medicine in Sport, vol. 23, no. 10, pp. 913-920, 2020.

[31] K. L. Piercy, R. P. Troiano, R. M. Ballard et al., "The physical activity guidelines for Americans," JAMA, vol. 320, no. 19, p. 2020, 2018.

[32] National Academies of Sciences, Engineering, and Medicine, Health and Medicine Division, Board on Health Sciences Policy, and Committee on Preventing Dementia and Cognitive Impairment, Preventing Cognitive Decline and Dementia: A Way Forward, National Academies Press, Washington, DC, USA, 2017.

[33] T. L. Neurology, "Pointing the way to primary prevention of dementia," Lancet Neurology, vol. 16, p. 677, 2017.

[34] E. You, K. A. Ellis, K. Cox, and N. T. Lautenschlager, "Targeted physical activity for older adults with mild cognitive impairment and subjective cognitive decline," Medical Journal of Australia, vol. 210, no. 9, pp. 394-395, 2019.

[35] G. Zheng, R. Xia, W. Zhou, J. Tao, and L. Chen, "Aerobic exercise ameliorates cognitive function in older adults with mild cognitive impairment: a systematic review and metaanalysis of randomised controlled trials," British Journal of Sports Medicine, vol. 50, no. 23, pp. 1443-1450, 2016.

[36] R. Jia, J. Liang, Y. Xu, and Y. Wang, "Effects of physical activity and exercise on the cognitive function of patients with Alzheimer disease: a meta-analysis," BMC Geriatrics, vol. 19, 2019.

[37] C. Groot, A. M. Hooghiemstra, P. G. H. M. Raijmakers et al., "The effect of physical activity on cognitive function in patients with dementia: a meta-analysis of randomized control trials," Ageing Research Reviews, vol. 25, pp. 13-23, 2016.

[38] T. Engeroff, T. Ingmann, and W. Banzer, "Physical activity throughout the adult life span and domain-specific cognitive function in old age: a systematic review of cross-sectional and longitudinal data," Sports Medicine, vol. 48, no. 6, pp. 1405-1436, 2018.

[39] F. Sofi, D. Valecchi, D. Bacci et al., "Physical activity and risk of cognitive decline: a meta-analysis of prospective studies," Journal of Internal Medicine, vol. 269, no. 1, pp. 107-117, 2011.

[40] K. Fabel and G. Kempermann, "Physical activity and the regulation of neurogenesis in the adult and aging brain," NeuroMolecular Medicine, vol. 10, no. 2, pp. 59-66, 2008.

[41] K. Fabel, "Additive effects of physical exercise and environmental enrichment on adult hippocampal neurogenesis in mice," Frontiers in Neuroscience, vol. 3, no. 50, 2009.

[42] S. Andrieu, N. Coley, S. Lovestone, P. S. Aisen, and B. Vellas, "Prevention of sporadic Alzheimer's disease: lessons learned from clinical trials and future directions," The Lancet Neurology, vol. 14, no. 9, pp. 926-944, 2015.

[43] E. de Bruin, P. Eggenberger, V. Schumacher, M. Angst, and N. Theill, "Does multicomponent physical exercise with simultaneous cognitive training boost cognitive performance in older adults? A 6-month rando-mized controlled trial with a 1-year follow-up," Clinical Interventions in Aging, vol. 10, pp. 1335-1349, 2015.

[44] N. Theill, V. Schumacher, R. Adelsberger, M. Martin, and L. Jäncke, "Effects of simultaneously performed cognitive and physical training in older adults," BMC Neuroscience, vol. 14, p. 103, 2013. 
[45] E. G. A. Karssemeijer, J. A. Aaronson, W. J. Bossers, T. Smits, M. G. M. Olde Rikkert, and R. P. C. Kessels, "Positive effects of combined cognitive and physical exercise training on cognitive function in older adults with mild cognitive impairment or dementia: a meta-analysis," Ageing Research Reviews, vol. 40, pp. 75-83, 2017.

[46] M. Borenstein, L. V. Hedges, J. P. T. Higgins, and H. R. Rothstein, "Introduction to meta-analysis," International Statistical Review, vol. 77, p. 478, 2009.

[47] N. C. Boa Sorte Silva, L. S. Nagamatsu, D. P. Gill, A. M. Owen, and R. J. Petrella, "Memory function and brain functional connectivity adaptations following multiple-modality exercise and mind-motor training in older adults at risk of dementia: an exploratory sub-study," Frontiers in Aging Neuroscience, vol. 12, 2020.

[48] M. A. Gregory, N. C. Boa Sorte Silva, D. P. Gill et al., "Combined dual-task gait training and aerobic exercise to improve cognition, mobility, and vascular health in community-dwelling older adults at risk for future cognitive Decline1," Journal of Alzheimer's Disease, vol. 57, no. 3, pp. 747-763, 2017.

[49] N. C. Boa Sorte Silva, D. P. Gill, A. M Owen et al., "Cognitive changes following multiple-modality exercise and mindmotor training in older adults with subjective cognitive complaints: the M4 study," PLoS One, vol. 13, Article ID e0196356, 2018.

[50] E. M. Shellington, M. Heath, D. P. Gill, and R. J. Petrella, "Long-term maintenance of executive-related oculomotor improvements in older adults with self-reported cognitive complaints following a 24-week multiple modality exercise program," Journal of Alzheimer's Disease, vol. 58, no. 1, pp. 17-22, 2017.

[51] S. C. McEwen, P. Siddarth, B. Abedelsater et al., "Simultaneous aerobic exercise and memory training program in older adults with subjective memory impairments," Journal of Alzheimer's Disease, vol. 62, no. 2, pp. 795-806, 2018.

[52] A. S. Chan, W. K. Cheung, M. K. Yeung et al., "A Chinese chan -based mind-body intervention improves memory of older adults," Frontiers in Aging Neuroscience, vol. 9, p. 190, 2017.

[53] N. T. Lautenschlager, K. L. Cox, L. Flicker et al., "Effect of physical activity on cognitive function in older adults at risk for Alzheimer disease," JAMA, vol. 300, no. 9, pp. 1027-1037, 2008.

[54] C. Fabre, J. Massé-Biron, K. Chamari, A. Varray, P. Mucci, and C. Préfaut, "Evaluation of quality of life in elderly healthy subjects after aerobic and/or mental training," Archives of Gerontology and Geriatrics, vol. 28, no. 1, pp. 9-22, 1999.

[55] S. Andrieu, S. Guyonnet, N. Coley et al., "Effect of long-term omega 3 polyunsaturated fatty acid supplementation with or without multidomain intervention on cognitive function in elderly adults with memory complaints (MAPT): a randomised, placebo-controlled trial," The Lancet Neurology, vol. 16, no. 5, pp. 377-389, 2017.

[56] J. P. T. H. Statistician, D. G. A. Director, P. C. G. Director et al., "The cochrane collaboration's tool for assessing risk of bias in randomised trials," BMJ, vol. 343, p. d5928, 2011.

[57] K. L. Cox, L. Flicker, O. P. Almeida et al., "The FABS trial: a randomised control trial of the effects of a 6-month physical activity intervention on adherence and long-term physical activity and self-efficacy in older adults with memory complaints," Preventive Medicine, vol. 57, no. 6, pp. 824-830, 2013.

[58] G. W. Small, D. H. S. Silverman, P. Siddarth et al., "Effects of a 14-day healthy longevity lifestyle program on cognition and brain function," The American Journal of Geriatric Psychiatry, vol. 14, no. 6, pp. 538-545, 2006.

[59] L. E. Middleton, M. I. Ventura, W. Santos-Modesitt, G. Poelke, K. Yaffe, and D. E. Barnes, "The mental activity and exercise (MAX) trial: effects on physical function and quality of life among older adults with cognitive complaints," Contemporary Clinical Trials, vol. 64, pp. 161-166, 2018.

[60] L. S. Nagamatsu, A. Chan, J. C. Davis et al., "Physical activity improves verbal and spatial memory in older adults with probable mild cognitive impairment: a 6-month randomized controlled trial," Journal of Aging Research, vol. 201310 pages, Article ID 861893, 2013.

[61] S.-Y. Cho, G.-H. Jahng, H. Y. Rhee et al., "An fMRI study on the effects of jaw-tapping movement on memory function in elderly people with memory disturbances," European Journal of Integrative Medicine, vol. 6, no. 1, pp. 90-97, 2014.

[62] H. Lavretsky, D. S. Khalsa, A. Leaver, H. Eyre, P. Siddarth, and H. Yang, "Changes in the functional brain connectivity and verbal memory performance following yoga or memory training in older adults with subjective memory complaints," Alzheimers \& Dementia, vol. 11, p. 896, 2015.

[63] L. Bherer, "Cognitive plasticity in older adults: effects of cognitive training and physical exercise," Annals of the New York Academy of Sciences, vol. 1337, no. 1, pp. 1-6, 2015.

[64] T. P. Ng, L. Feng, M. S. Z. Nyunt et al., "Nutritional, physical, cognitive, and combination interventions and frailty reversal among older adults: a randomized controlled trial," The American Journal of Medicine, vol. 128, no. 11, pp. 1225-1236, 2015.

[65] D. E. Barnes, W. Santos-Modesitt, G. Poelke et al., "The mental activity and exercise (MAX) trial: a randomized controlled trial to enhance cognitive function in older adults," JAMA Internal Medicine, vol. 173, no. 9, pp. 797-804, 2013.

[66] R. Bhome, A. J. Berry, J. D. Huntley, and R. J. Howard, "Interventions for subjective cognitive decline: systematic review and meta-analysis," BMJ Open, vol. 8, Article ID e021610, 2018. 